\title{
Supporting Multi-agent Coordination and Computational Collective Intelligence in Enterprise 2.0 Platform
}

\author{
Seddik Reguieg* and Noria Taghezout \\ LIO Laboratory, Dept. of Computer Science, University of Oran 1, Ahmed Ben Bella, BP 1524, El \\ Mnaouer, Oran (Algeria)
}

Received 24 January 2017 | Accepted 24 June 2017 | Published 18 July 2017

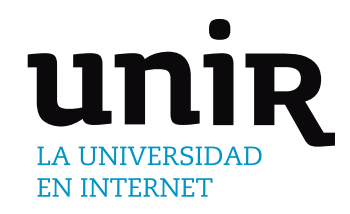

\section{KEYWORDS}

In this paper, we propose a novel approach utilizing a professional Social network (Pro Social Network) and a new coordination protocol (CordiNet). Our motivation behind this article is to convince Small and Medium Enterprises managers that current organizations have chosen to use Enterprise 2.0 tools because these latter have demonstrated remarkable innovation as well as successful collaboration and collective intelligence. The particularity of our work is that is allows employer to share diagnosis and fault repair procedures on the basis of some modeling agents. In fact, each enterprise is represented by a container of agents to ensure a secured and confidential information exchange between intra employers, and a central main container to connect all enterprises' containers for a social information exchange. Enterprise's container consists of a Checker Enterprise Agent (ChEA), a Coordinator Enterprise Agent (CoEA) and a Search Enterprise Agent (SeEA). Whereas the central main container comprises its proper agents such as Selection Agent (SA), and a Supervisor Agent (SuA). JADE platform is used to allow agents to communicate and collaborate. The FIPA-ACL performatives have been extended for this purpose. We conduct some experiments to demonstrate the feasibility of our approach.

\author{
Collaboration, \\ Collective Intelligence, \\ Coordination Protocol \\ (CordiNet), Enterprise \\ 2.0, Pro Social Network, \\ Multi-Agent System \\ (MAS).
}

DOI: $10.9781 /$ ijimai.2017.07.001

\section{INTRODUCTION}

$\mathrm{R}$ ECENTLY, an ecosystem of employees, partners, suppliers, and customers has begun to proliferate by using web 2.0 technologies, to develop capabilities by collectively generate, share and refine business knowledge. This ecosystem is named as an enterprise 2.0 collaboration platform [1].

These kinds of platforms provide enterprises with new models and tools for an emergent collaboration and co-creation to assure and to harness collective intelligence [2], [3], [4].

In addition, the mobilization of collective intelligence in enterprises 2.0 platforms encourages teamwork and knowledge sharing. The adoption of web 2.0 technologies as new collaborative management practices allows turning the emphasis on the development of human capital such as the knowledge, experience and skills of members and on the development of social capital - like relationships, communities and norms that facilitate collective action and collaborative behavior.

To be effective, our work covers a wide range of goals and tasks in a more sophisticated way to lead the world and the current industry to a new win-win spirit as an agreement whereby each enterprise is also concerned with the interests of the other in an equally favorable to its own interests. In other words, it is an agreement that increases the earnings of each.

Enterprise 2.0 supports the informal organization as a set of

\footnotetext{
* Corresponding author.

E-mail address: rseddiko@gmail.com
}

technologies i.e. social networks [5]. These social networks are combined with other resources as areas of collaboration, connection and communication to improve the organization of knowledge, skills of users and encouraging collaboration and innovation [6], [7]. Furthermore, the ability of a social group to develop its human capital has become a brand of collective intelligence within the meaning of [8], [9]: distributed intelligence everywhere, constantly valued, coordinated in a real time, resulted in a "full" mobilization of skills.

Thus, according to the study that is provided in [10], a social network is defined as a set of relations of a specific type (e.g., collaboration, support, consulting, control, or influence) between a set of actors. Each actor in the network is required to create links with other actors; analysis of these links can predict the characteristics of the actors or the appearance of links between them.

Furthermore, social network can play an important role in supporting different types of decision making, as they provide their users various forms of support, ranging from instrumental to the emotional and informational. The synergy of these themes provides an innovative and unique perspective on the actual process of decision-making within social network.

An effective decision-making is often based on the control of information over time. This action aims to capture the current state of the most important pieces of information that are updated in different ways. In Enterprise 2.0 platform, employers are looking for the right information at the right time under confidentiality and security. However, employers don't have all necessary information such as diagnosis or failure solution procedures. This information can be found intra enterprise (coordination), or inter enterprise (collaboration). To ensure exchange knowledge intra enterprise we must first ensure coordination. 
The coordination can be expressed by different techniques, which are summarized in: coordination languages, coordination algorithms, and coordination protocols. Several works have appeared in the field of coordination [11], [12], [13], which propose solutions that can provide consistency in production systems. Among those works, we can cite [14]; this approach is a centralized one. It is composed of a single agent which is responsible of the coordination system. This is on the one hand but on the other, several architectures that are [15] based on the distributed aspect were developed in order to provide a large number of interactions.

This work contributes within several principles and technicalities to build an enterprise 2.0 platform and to achieve collective intelligence via information sharing among trusted contacts in several enterprises.

Our platform consists on two levels: a collaborative environment, that allows users to exchange knowledge and information inter employers in different enterprises using their relations in social network, and coordination environment for exchanging knowledge and information intra enterprise which is based on a multi-agent system and coordination protocol. The motivation of our platform's components is to make an efficient decision by offering effective diagnosis and failure solution procedures with a secured and confidential support.

In this article, we focus on the study of two basic components of the framework: the professional social network (Pro Social Network) and the coordination protocol (CordiNet). Pro Social Network is dedicated to companies that want to share their knowledge and expertise on the industrial diagnosis. CordiNet represents a coordination protocol that manages interactions between agents.

After a brief introduction that describes the context. Section 2 of this paper discusses some approaches of enterprise 2.0, collective intelligence, and multi-agents systems, emphasizing their strengths and limitations. The proposed approach is being presented in section 3 of the article. Herein, Pro Social Network as well as CordiNet are described. In Section 4, the most important performatives of the protocol are provided with some experiments that have been conducted with the proposed protocol. Finally, a conclusion regarding the research works as well as future works are mentioned in Section 5.

\section{BACKGROUND}

This section presents an overview of related work (social networks, multi-agents systems and enterprise 2.0) and presents our major contribution as a continuation of this background.

\section{A. Social Networks and Multi-agents Systems}

Many studies have integrated agents in social networks in different ways. In this section, we concentrate on the most relevant works that influence our research.

Authors in their literature review on [16] internet based collaboration tools have mentioned that online collaboration tools are divided into 2 classes; the first one regrouped some tools that are based on internetbased technologies while the second class comprised the tools that are based on Web 2.0 technologies. These latter provides a support for a beneficial collaboration. Some well-known examples of these communication tools are wikis, blogs, forums, RSS feeds, community chats and social networking. Collaboration 2.0 has been initiated by the use of Web 2.0 tools. This new form of collaboration allows a robust real time collaboration between dispersed participants as it was well explained in the work [17].

More precisely, in the work that was presented in [17], the domain experts were given a collaborative WEB interface in order to save and capitalize their business knowledge as business rules through an agentbased platform. Furthermore, domain ontology has been developed in order to generate the business model corresponding to the enterprise and check the consistency of business rules. The modeling was based on some agents such as Expert agent, Evaluator agent, Translator and Security agents. The main objective was to increase the execution speed of processes and effective response [17].

Among the early works on software agents supporting social networking system, the papers [18], [19] and [20] are particularly relevant.

In paper [18], author presented a multi-agent system that implemented a distributed social network system. By using such system, users are considered as the sole owners of the information they provide when they address privacy. The main particularity of this system is that users are represented by agents that both mediate access to private data and proactively negotiate with other agents in order to extend their user's social network. A distributed connection discovery algorithm is also presented. This algorithm is developed to suggest connections to the users for constructing a social network through the information that is stored in their profile. This constitutes the main advantage of this approach. The major limitation is that the privacy is not guaranteed by only the fact that the users can specify which data shall be used in order to rich their social network especially when we deal with friends of friends.

The study that was provided in [20] is quite different from other works which supported the social networking. This is due to the key points that were addressed in the corresponding paper. Our deeper review of the content gives us a precise point of view of the advantages of the use of enterprise 2.0 in project management such as the sharing of information and expertise between operators and senior managers and time flexibility.

Furthermore, this research offers a good list of examples of well-known companies that opened their road to success by promoting the web 2.0 model in their projects and activities related to their organization.

In the work presented in [19], a simple framework has been proposed to help understand the collaboration that was offered by Web 2.0 technologies.

The study was based on the development of a Web-based Decision Support System (DSS) which included five agents: Analyzer agent, Proposal agent, Resource agent, production agent and Coordinator agent. These latter were used to examine how human participants can create, share and exchange experiences or diagnosis on resources failures which each other to have new ideas or useful information for the decisionmaking process. The limitation of this work consists in improving the communication and collaboration between the human operators and integrating a case base reasoning to take benefit from past experiences.

\section{B. Research on Enterprise 2.0}

As defined in [20] [21], Enterprise 2.0 or E2.0 is represented as a set of organizational and technological approaches to implement new organizational models that are based on mass participation, technologyoptimized collaboration, knowledge sharing, and exploitation of professional social networks.

From our review on enterprise 2.0 notion and its application in industrial domain, we find that this technology has encountered various difficulties like any other technological innovation (technological, organizational, cultural, social...) [22].

It seems that the more imminent challenge is the compatibility of Web 2.0 with the enterprise culture and existing technological infrastructure more precisely with the manager's attitude. As well explained in [20], the acquisition of Web 2.0 tools is not enough to say that a company is an Enterprise 2.0. Corresponding managers and employers need to be motivated. The adoption of Enterprise 2.0 concept is conditioned by culture and certain attitudes. 
The review of the current proposals in the literature shows that the work presented in Schauer et al. [23] offers very interesting features; Authors introduced open-source enterprise 2.0 tools to support knowledge workers in the execution of cognitive tasks and information sharing. They also presented a study of the growing market for enterprise 2.0 systems using an open source license. In their study, they used a set of 97 individual features and criteria that are grouped around the central functionalities of communication, coordination and collaboration, in order to analyze the suitability of a representative sample for the average knowledge worker.

Even more, among the basic functionalities of enterprise 2.0 platforms, we cite electronic collaboration. In this context, a new study was described in [24], which can be considered as a real contribution for a better understanding by providing an overview of the market for E-Collaboration software packages. Based on the classification that was done in [24], five system classes have been identified to characterize the market for Collaboration systems.

The first identified class regrouped Everyday systems which are used continuously by the group as primary systems to support everyday activities. Such systems provide basic features to support all three processes of communication, coordination and collaboration.

Integrated systems were considered as the second class of systems that share many features (e.g. E-Mail, calendars, address books and task lists) with everyday systems, but their constituent characteristic is the support of synchronous communication.

Another class of coordination systems has been identified. As mentioned in [24], this class comprises a total of 24 systems which focus on group coordination. Particularly, task coordination systems and process coordination systems are constituents of this class.

A set of 13 systems are grouped in a class named meeting systems that support online and video conferences. The systems are based on extensive synchronous communication featuring such as text chat, audio and video communication.

Regarding these 4 classes, we can say that our approach belongs to the subclass that regroups cases converging from one class to another, such as the systems being at the convergence of the coordination systems to the integrated systems.

As clearly explained in [24], the systems integrating project features are quite similar to coordination ones. The collaboration and coordination processes are supported by the coordination systems, whereas the integrated systems add the communication aspect that we find in the WEB 2.0 technologies integrated in our project. In addition to their project management capabilities, integrated systems as we see them are systems that provide a set of features ranging from a simple to knowledge management functionality regarding to the organization of an enterprise. This category of systems easily adheres to the use of WEB 2.0 tools augmented by a set of additive project coordination functions.

Whereas $\mathrm{Xu}$ et al. [25] presented a survey on asynchronous collaboration tools that allow users to collaborate at different times. They structured their features in four major functional categories such as: communication, information sharing, electronic calendar and project management.

Furthermore, authors in [26] advocated the idea that enterprise collaboration must evolve towards a new paradigm in which knowledge workers are considered as co-producers of information, software services and applications without involving IT departments. Their prototype named EzWeb consisted on an implementation of an open Enterprise 2.0 collaboration platform that empowered its users to co-produce and share instant application.

In the study that was provided in [27], authors proposed a model of Enterprise 2.0 as a mash up developer which is supported in searching for assistance from developers owning specific knowledge, according to typical collaboration patterns. Their study focused on collaborative development in the Enterprise 2.0 contexts which include Web APIs for sharing Web sites, information about developers based on a specialization of ontology and relationships among developers and Web APIs.

In other works such as [35], authors were interested by sharing and managing knowledge in enterprise 2.0 platform. In this paper, author presented a model integrating the interpersonal network in enterprise 2.0. They took the knowledge governance as a regulator variable between enterprise knowledge system and its environment, a relationship between the performance of knowledge collaboration and structure entropy has been also built. In their paper, they constructed the self-organization concept model and logistic process analysis model of knowledge system in enterprise 2.0 environment, which are to explore the evolution pattern of knowledge collaboration system in favor of the organization mechanism design for enterprise knowledge governance. However, in enterprise 2.0 platform, sharing knowledge must be done under confidentiality and security which is not describe in their work. Authors mentioned also that their work lacks presumption appropriate control parameters and formulation of governance structure and strategies.

\section{Our Contribution and Comparison with other Works}

In current small and medium enterprises that exist in our country, collaboration became a very important way to proliferate or succeed. These Organizations used to ensure a lot of activities around blogs and wikis with a focus on collaboration and sharing of knowledge. Managers are usually encouraging employees to be connected with Facebook or LinkedIn social network but they don't feel ready to consider a project based on Enterprise 2.0.

Our motivation behind this work is to convince senior managers that Enterprise 2.0 is a real solution which allows exploiting the WEB 2.0 technologies.

In collaborative platforms or social networks, Web servers are constantly overloaded with client requests. An Enterprise 2.0 platform is essentially based on a web server that provides several services namely: internal tools and applications such as online ERP, monitoring, collaboration and coordination applications. As a result, services are multiplying in response to the number of companies and users who are members benefiting from all the functionalities offered by this platform.

To simplify the work of the web server, we propose a multi-agent system in order to perform complex tasks while ensuring the security and integrity of each company's data. By sharing tasks with the web server, which will only be dedicated to displaying and processing replies to users' requests, the multi-agent system takes over and searches for information in each database of each company, in an invisible way to the customer.

The originality of our approach lies in the suggested coordination protocol (CordiNet) which regroups a set of primitives that are deployed by realizing extensions of the performatives of the FIPA-ACL.

Moreover, the Supervisor agent (SuA) of the central container plays a very important role; it makes it possible to efficiently manage the work of every coordinator agent of the enterprise. The search for information in our platform is supported by the selection agent (SA) which executes a search orientation algorithm according to the semantics of the request and its priority in the system.

In Table 1, we present a comparison between some related works and ours. We just sort the items by using '+' to indicate that the option exists and '-' to indicate that the option doesn't exist. We put our approach in the bottom of Table 1. Some indications are given with the Table1 in order to clarify some criteria that are used in the comparison.

\section{A: Enterprise 2.0}

B: Social network 


\section{C: Coordination}

D: Multi-Agents System

E: Interaction Protocol

F: Ontology

G: Learning Aspect

$\mathrm{H}$ : Information retrieval mechanism

I: Recommendation System

J: Knowledge sharing

As we can see in Table 1, no work has introduced the concept of multi-agents and protocols in enterprise 2.0 platform.

TABLE I. Comparison between Some Related Works and our Approach

\begin{tabular}{|c|c|c|c|c|c|c|c|c|c|c|}
\hline Works $^{1}$ & $\mathrm{~A}$ & $\mathrm{~B}$ & $\mathrm{C}$ & $\mathrm{D}$ & $\mathrm{E}$ & $\mathrm{F}$ & $\mathrm{G}$ & $\mathrm{H}$ & $\mathrm{I}$ & $\mathrm{J}$ \\
\hline 1 & + & + & + & - & - & - & - & - & - & + \\
\hline 2 & - & + & + & - & - & - & - & - & - & + \\
\hline 3 & - & + & + & - & - & - & - & + & - & + \\
\hline 4 & + & + & - & - & - & + & - & - & + & + \\
\hline 5 & + & + & - & - & - & + & - & + & - & + \\
\hline 6 & + & - & + & - & - & - & - & & - & + \\
\hline 7 & + & + & + & + & + & - & - & + & + & + \\
\hline
\end{tabular}

${ }^{1}$ Works: 1: Schauer et al. [23], 2: K. Riemer [24], 3: Xu et al. [25], 4: J. Soriano et al. [26], 5: D. Bianchini et al. [27], 6: X. Yun et al. [35]; 7: Our work.

Our contribution is to integrate multi-agents system into enterprise 2.0 platforms and more precisely into its social network.

An Enterprise 2.0 platform consists of several subsystems and components that are all connected to a Web server. The increase in the number of companies adhering to our platform implies an increase in the number of users; this could either cause a system crash or generate no response because the Web server cannot handle all requests (multiple users, multiple components). To avoid this, we have introduced a multi-agent system and an interaction protocol to perform a distributed processing which does not necessarily includes the web server, such as the search for information (in our case, the search for Industrial diagnostics).

In the next section, we describe and motivate the problem on which we will focus in the development of a collaborative platform that also supports the coordination of its agents.

\section{PROPOSED APPROACH}

The business model of enterprises has changed from an old one which is based on commands and information management to a new one which integrates a new paradigm of collaboration that is based on the WEB 2.0 technologies. In Our approach, we find two different types of enterprises information flows namely: external flow which will describe collaboration environment and internal flow for coordination environment. Figure 1 presents our platform that consists on several spaces which allow enterprises to collaborate, coordinate and control their internal or external flows. In this paper, we will focus and develop the collaboration and coordination space.

\section{A. Pro Social Network Design}

In the first environment, we implement a social network as a support to provide companies of new models and tools for collaboration, participation and co-creation.

This collaboration is established between the various participants such as company employees, customers, suppliers, experts with the ability to create groups that can contain these profiles.

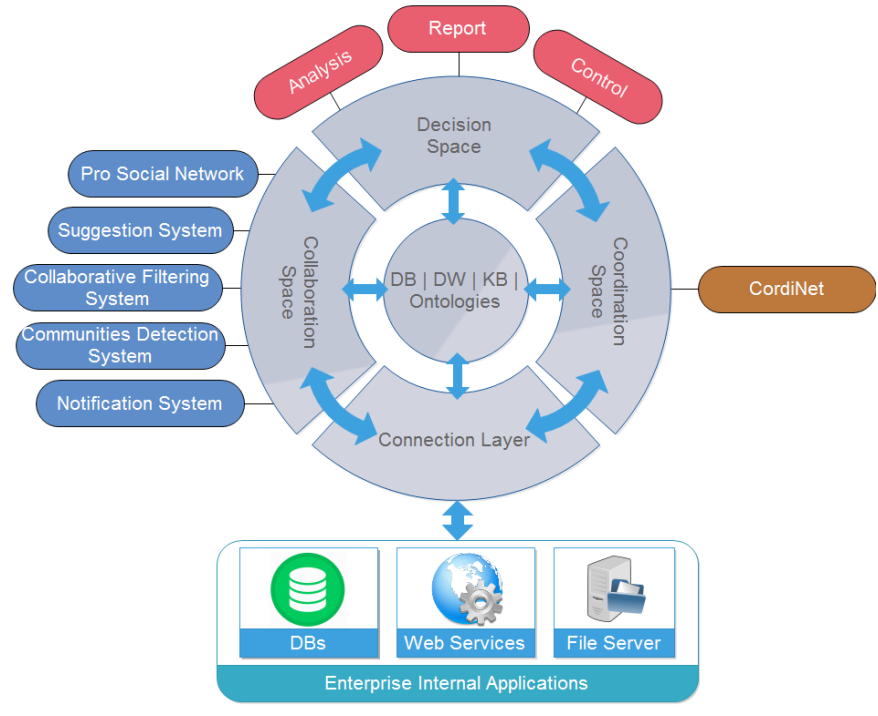

Fig. 1. Architecture of our Enterprise 2.0 platform.

The profile is given throughout an interface that represents a user, group or company on our network. This latter corresponds to an identity that is established on the network. It may be a reflection of a real or virtual identity. The virtual identity means that any user can create an account, in this case user does not belong to a company which is adhered to our platform, and we cannot identify his real identity, it is said Freelancer while the real identity means that the account is given to users who belong to a company that adheres to our platform, an identity verification must be done in the administrative space of this company, the HRD account validates the creation of this account.

Our collaborative environment ensures the allocation of a digital professional identity to different profiles. It allows the establishment of the collaboration while enriching the relationship between the different profiles; the main objective behind this is to maintain the continuity of the professional links.

To fully enhance the collaborative environment, we have introduced a feature that allows the user to adopt good practices during knowledge sharing and participation in collaborative work.

Figure 2 presents an example of exchanging information between actors. For example, let's us consider a simple user named as User $\mathrm{X}$, this latter is in relationship with some other users of the same or different enterprise. Thus, he can share information on his wall, enterprise wall or his groups' wall.

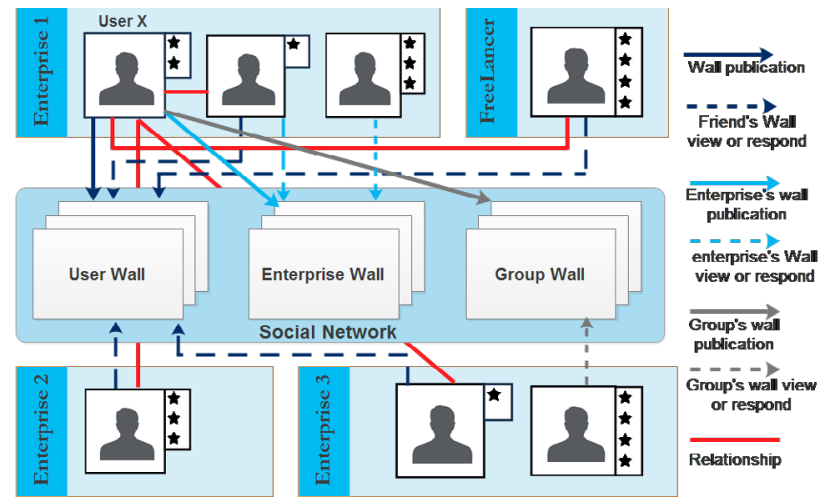

Fig. 2. Information's exchange in our social network.

Our social network disposes some features for enterprise management and users like: profiles, expert base, relationship 
management, flow of activities and foremost, asynchronous and synchronous communication.

In what follows, we present the general structure and features that characterize a user's account (Figure 3).

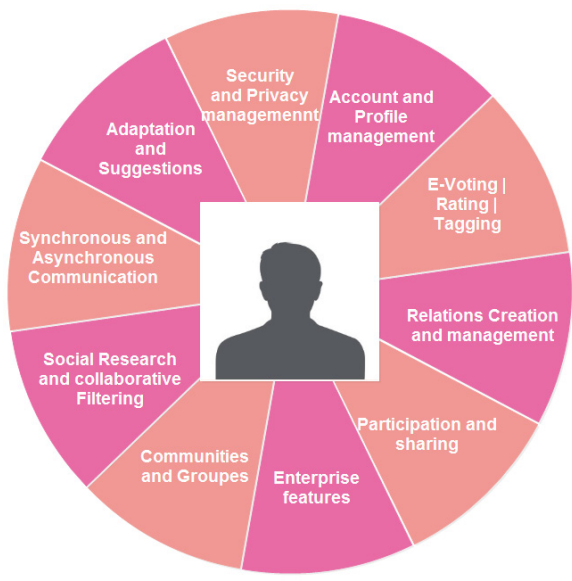

Fig. 3. User's account features.

More precisely, to access our platform, we provide two ways ; (i) by creating an account and enriching it, (ii) by using APIs that are offered by social networks (Google+, Facebook, etc) in order to retrieve the existing profiles from these networks.

Our collaborative environment allows participants to share and exchange diagnosis under confidentiality and security. Each enterprise can manage its employers in our platform, and each user can share diagnosis with its collaborators (different enterprise) or colleagues (same enterprise). By using a catalog, this latter can set, update and delete diagnosis and failure solution procedures in an easy way.

To give more confidentiality for user intervention on the use of catalog, many options are given as below:

1. Me only: It means that user is the only one who can see this publication

2. Colleague: It means that a colleague who is in relationship with this user can get this diagnosis.

3. Collaborator: it means that collaborators who are in relationship with this user can get this diagnosis.

4. Freelancers: it means that the freelancers who are in relationship with this user can get this diagnosis

5. Open: all users that are connected to the platform can get this diagnosis.

User can use a customized type to combine between confidentialities in case of need (except for Me only and Open) eg. Confidentiality $=$ colleague and collaborators, Confidentiality $=$ colleagues and freelancers, Confidentiality $=$ collaborators and freelancers.

When user searches an industrial diagnosis of any machine, the result must respect diagnosis' confidentiality that is established by their editors.

As it can be seen a lot information are exchanged during the treatments, it's why we opt to use agents as modeling entities in order to control the whole system and we develop a coordination protocol for this task.

\section{B. CordiNet Design}

In the second environment, we want to set up a coordination environment (CordiNet) to exchange knowledge and information between employers of the same enterprise. The goal is to make the system more secure and confidence, reactive and produce a quick execution. In this section we present our coordination environment as a multi-agent system and the corresponding coordination protocol.

Our approach uses reactive and cognitive agents which need an elaborated language to be able to exchange messages. However, a structural format of a message is not sufficient in itself to be able to formalize all the conversations between agents. The protocol concept was introduced to support such conversations, allowing speech acts. We can define coordination protocols as shared conventions on messages that are exchanged by agents working together in a coordinated manner. More protocol is effective; less information needs to be transmitted and less time is spent in the communication as mentioned in [28].

\section{Agent-based Modeling}

The use of a multi-agents system that is opened in an application for Internet offers three main advantages: scalability, stability and load balancing.

The advantages of Multi-agents system (MAS) such as autonomy, adaptability, knowledge level, learning aspects and availability of interaction protocols have encouraged us to adopt them in our platform to balance overload the web server [29].

Our multi-agent architecture is developed as a blackboard behind the Web server. The proposed model mainly consists of two types of containers; enterprise container which controls internal enterprise flow and central main container which controls external flow of our collaborative environment (see Figure 4).

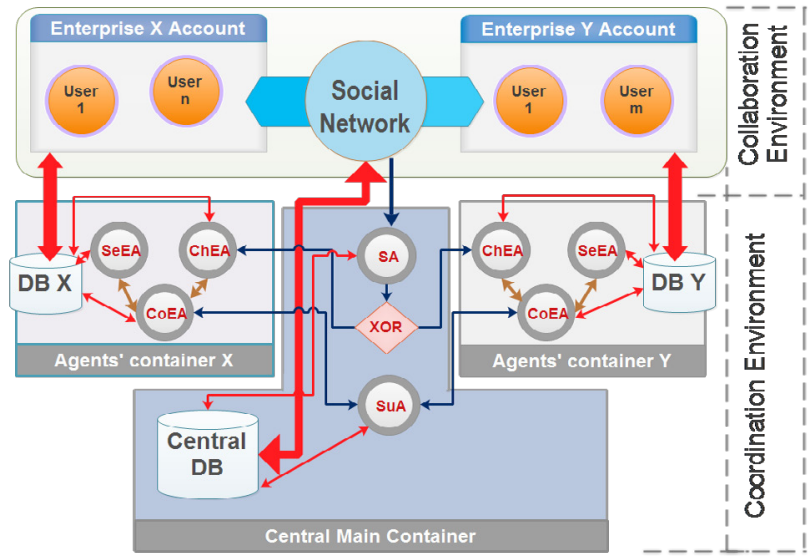

Fig. 4. Architecture of our Multi-agent system to support our social network.

Each enterprise that is connected to our platform is represented by a container. In fact, every enterprise Container consists of three components: Checker Enterprise Agent (ChEA), Search Enterprise Agent (SeEA), and Coordinator Agent Enterprise (CoEA). The central main container consists of two components: Selection Agent (SA), and Supervisor Agent (SuA).

We have also developed a coordination protocol ensuring the exchange of messages between internal flow and external flow. The $\mathrm{SuA}$ has a learning aspect. This mechanism facilitates decision-making in the system and requires less execution time. Coordination in multiagent systems can be performed in a centralized manner in which circulation of information between enterprises is assured by the central main container's agents.

As we have previously described, we had used two types of container: enterprise's container and central main container.

1. Enterprise Container: : It is a container that regroups agents that control internal flows in a given enterprise, when an enterprise adheres to our platform then enterprise's container's agents are created by Supervisor Agent (SuA) of central container. These 
agents are described as follows:

- Checker Enterprise Agent (ChEA): It is a reactive agent which detects if a new diagnosis is required by enterprise user.

- Search Enterprise Agent (SeEA): It is a cognitive agent which searches if diagnosis exists in enterprise's database and returns it.

- Coordinator Enterprise Agent (CoEA): It is a cognitive agent which works with the Central Coordinator Agent in order to receive diagnosis' request and processing. It verifies confidentialities and undesired messages. The internal architecture of CoEA is illustrated in Figure 5.

2. Central Main Container: includes 2 agents that control external flows coming from different enterprises such as exchanging of diagnosis namely:

- Selection Agent (SA): It is a reactive agent which selects and directs the diagnosis' request to the corresponding agent enterprise according to any user of our social network.

- Supervisor Agent (SuA): It is a cognitive agent which controls enterprises' containers, by creating and supervising certificated agents. In addition of its specific role of supervision, this agent executes some tasks such as verification of confidentiality, detection and filtering of undesired messages and control of all messages. The internal architecture of SuA is illustrated in figure 6 .

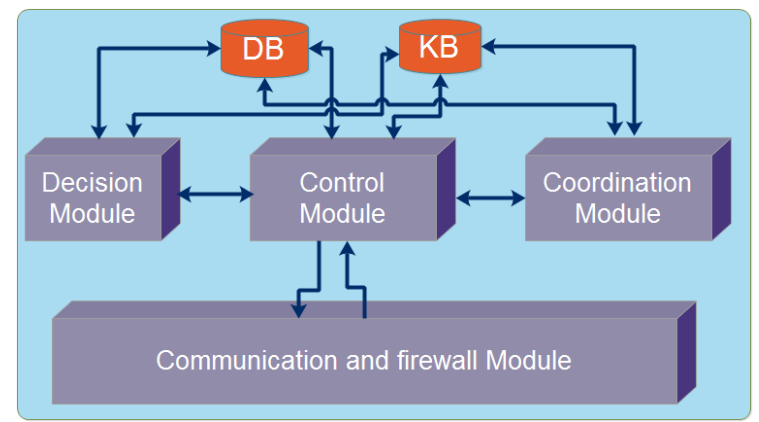

Fig. 5. Internal architecture of COordinator Enterprise Agent (CoEA).

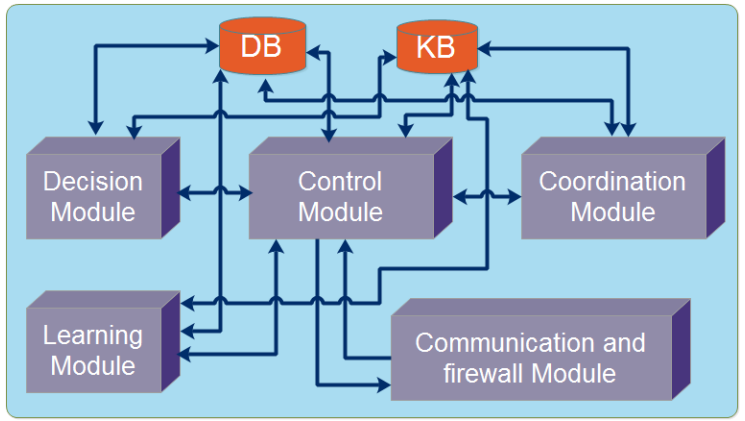

Fig. 6. Internal architecture of Supervisor Agent (SuA).

\section{The Proposed Protocol}

An agent sends a message in two cases: (i) When receiving a message that needs an answer; and (ii) When executing an action and needs information. Each message sent of our protocol is designed using the language communication FIPA-ACL [30].

In our coordination protocol, each type of agent has some behavior classes that are ready to use. Classes contain a set of methods in order to monitor each step of the protocol. They are called when specific messages are received or are to be sent and must be treated in order to adapt the protocol to its context of use. In what follows, we give a general description of our coordination protocol.
1. The sending of messages: An agent sends a message in both cases: Following receipt of a message that requires a response or when performing an action.

2. Structure of a message that is sent: Each message which is sent in our protocol is designed using the communication language FIPAACL [30]. In our MAS, each message has the following syntax:

- Content Object: Content of message.

- Protocol: Protocol used to exchange ACL messages.

- Conversation-id: Conversation identifier (expression) that is used for the following acts of communication.

- Reply-with: The term used by the agent as to identify this message.

- Receiver: the name of the agent who receives the message.

- Sender: the name of the agent who sends the message.

3. Secure communication: In a replay attack gained knowledge of data content is used to modify data that has been transported before to acquire unauthorized information. In case of a spoofing and masquerading attack, we use certificated messages where each agent has a communication and firewall module. Firewall module helps to eliminate undesirable messages by identifying a digital signature.

4. The rules: The rules define the possible message exchange between agents under certain conditions. This representation is taken from [31] and appears to be most suitable for the description of our protocol due to its ease of writing and reading, and for its rigorous notation that facilitates coding. The upper part of the rule 1 defines the sending of a message from $\mathrm{X}$ to $\mathrm{Y}$. The lower part defines actions ( 1 ... an) that can be executed by $\mathrm{Y}$, after that, $\mathrm{Y}$ sends a response to $\mathrm{Z}$ if the condition $(\mathrm{C})$ which is expressed at the right of the rule is verified ( $\mathrm{Z}$ is optionally $\mathrm{X}$ ).

$$
\text { NameofRule } \frac{X \rightarrow \text { Message } \rightarrow Y}{A 1, A 2, \ldots, \text { An }-\operatorname{Re} \text { sponse } \rightarrow Z} C
$$

5. Classes of our protocol: We distinguish 3 classes that composed our protocol in order to manage different situations for each agent. Some of the classes of our protocol represent finite state machines, in fact with state and transitions.

- panneUserInitiator Class : It is used by CoEA when there is a search of a diagnosis launched by user. Figure 7 shows the finite state machine of this class and Table 2 gives its description.

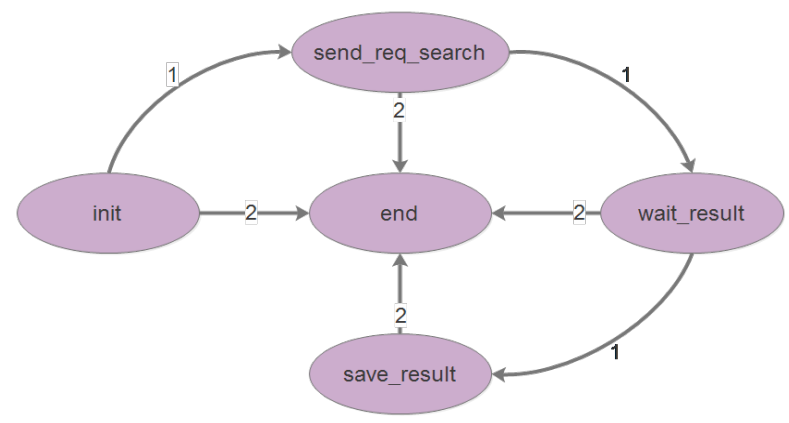

Fig. 7. Finite state machine of panneUserInitiator. 
TABLE II. PANNEUSERINITTIATOR's States AND Transitions DESCRIPTIONS

\begin{tabular}{|c|c|c|}
\hline State $^{1}$ & Description & Transition \\
\hline 1 & $\begin{array}{c}\text { Initialization of } \\
\text { protocol }\end{array}$ & $\begin{array}{c}1: \text { Preparing information search } \\
2: \text { Error of initialization }\end{array}$ \\
\hline 2 & $\begin{array}{c}\text { Sending information } \\
\text { search }\end{array}$ & $\begin{array}{c}1: \text { Information search request send } \\
2: \text { Error of sending }\end{array}$ \\
\hline 3 & $\begin{array}{c}\text { Waiting result of the } \\
\text { searched information }\end{array}$ & $\begin{array}{c}\text { 1: Result received with success } \\
2: \text { Error of waiting }\end{array}$ \\
\hline 4 & $\begin{array}{c}\text { Saving result and } \\
\text { forwarding feedback }\end{array}$ & $\begin{array}{c}1: \text { Operation succeed } \\
2: \text { Error of saving or forwarding }\end{array}$ \\
\hline 5 & End of protocol & None \\
\hline
\end{tabular}

${ }^{1}$ States: 1 : init, 2: send_req_search, 3: wait_result, 4: save_result, 5 :end.

- panneUserResponder Class: It is used by CoEA to send the diagnosis after receiving request from panneUserInitiator. This class replaces the user behavior while respecting its confidentialities put in place.

- panneCoordinatorCentral Class: It is used by Supervisor Agent (SuA) for receiving a diagnosis's request, collecting available CoEA, sorting CoEA list by learning mechanism, sending diagnosis requests to list of CoEA, receiving diagnosis and solutions. Figure 8 shows the finite state machine of this class while Table 3 gives its description.

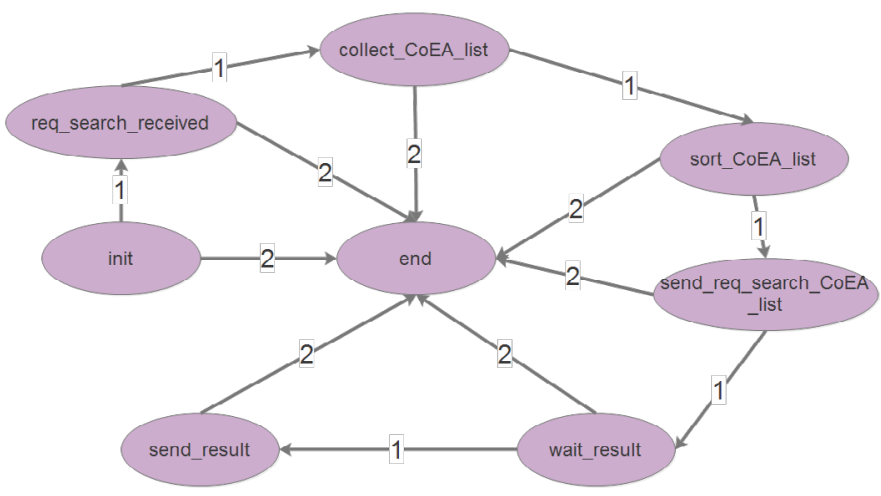

Fig. 8. Finite state machine of panneCoordinatorCentral.

TABLE III.

Panne Coordinator Central's States and Transitions Descriptions

\begin{tabular}{|c|l|l|}
\hline State $^{1}$ & \multicolumn{1}{|c|}{ Description } & \multicolumn{1}{c|}{ Transition } \\
\hline 1 & $\begin{array}{l}\text { Initialization of } \\
\text { protocol }\end{array}$ & $\begin{array}{l}\text { 1: Initialization request waiting } \\
\text { 2: Error of initialization }\end{array}$ \\
\hline 2 & $\begin{array}{l}\text { Waiting information } \\
\text { search request }\end{array}$ & $\begin{array}{l}\text { 1: Waiting of request information search } \\
\text { 2:Error of waiting }\end{array}$ \\
\hline 3 & $\begin{array}{l}\text { Collecting list of } \\
\text { active CoEA }\end{array}$ & $\begin{array}{l}\text { 1: Success of collect } \\
\text { 2: Error of collect }\end{array}$ \\
\hline 4 & $\begin{array}{l}\text { Sorting CoEA list } \\
\text { with search strategy } \\
\text { max uniform cost }\end{array}$ & $\begin{array}{l}1: \text { Establishing search strategy to sort } \\
\text { CoEA list to get optimal solution } \\
\text { 2: Error strategy or sorting list }\end{array}$ \\
\hline 5 & $\begin{array}{l}\text { Sending information } \\
\text { search to CoEA list }\end{array}$ & $\begin{array}{l}\text { 1: Send information search request to } \\
\text { CoEA succeed } \\
\text { 2: Error sending }\end{array}$ \\
\hline 6 & Waiting results & $\begin{array}{l}\text { 1: Waiting until results received } \\
\text { 2: Error of waiting }\end{array}$ \\
\hline 7 & $\begin{array}{l}\text { Sending results to } \\
\text { CoEA }\end{array}$ & $\begin{array}{l}\text { 1: Sending results succeed } \\
\text { 2: Error of sending results }\end{array}$ \\
\hline 8 & End & None \\
\hline
\end{tabular}

${ }^{1}$ State: 1: init, 2: req_search_received, 3: collect_CoEA_list, 4: sort_CoEA_ list, 5: send_req_rearch_CoEA_list, 6: wait_result, 7: send_result, 8: end.

\section{Communication between Agents}

Finding diagnosis and failures solutions bring us to information seeking context. This notion is an important part of our framework. To describe how diagnoses are founded, AUML sequence diagram (Figure 9) and UML sequence diagram (Figure 10) are given.

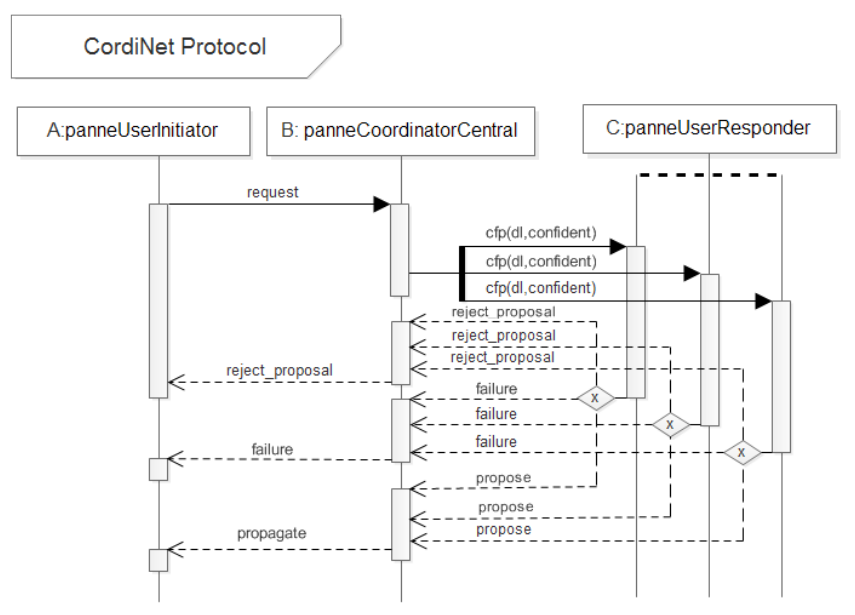

Fig. 9. AUML sequence diagram of CordiNet Protocol.

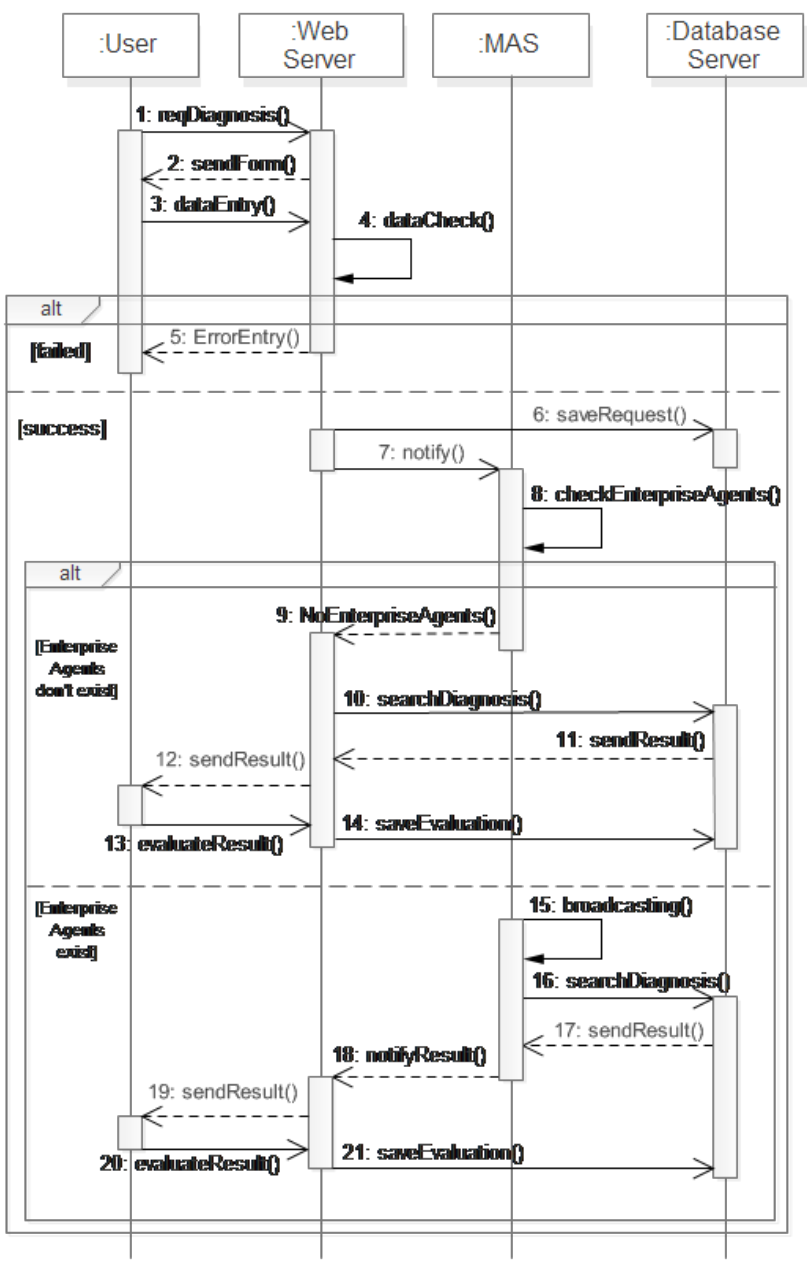

Fig. 10. Form for publishing and adding a diagnosis to a catalog.

In the sequence diagram that is presented in Figure 9, we distinguish three actors: a panneUserInitiator which corresponds to the initiator agent (CoEA of the applicant user enterprise), a panneCoordinatorCentral which corresponds to the Supervisor agent 
of main container $(\mathrm{SuA})$, and panneUserResponder which corresponds to all participants agents (all CoEA of all enterprises).

After the reception of a request from the panneUserInitiator to panneCoordinatorCentral, a cfp message is sent to all panneUserResponder with a deadline and confidentialities.

We distinguish three types of response:

- reject proposal if time of response is out.

- propose if a solution is found then this set of solution is propagated to panneUserInitiator.

- Failure in others cases (agent error, database error, etc...).

Figure 10 shows interaction between user, webserver, multi-agents system and database server. The internal process of MAS actor is given by the previous AUML (Figure

\section{General Process of Information Seeking}

To describe how information is sought we adopted the description from [32] and defined information seeking task as: the act of seeking information as a consequence of a need to satisfy some goal. Furthermore, search process has been studied extensively [33], [34]. Figure 11 summarizes our process of information retrieval.

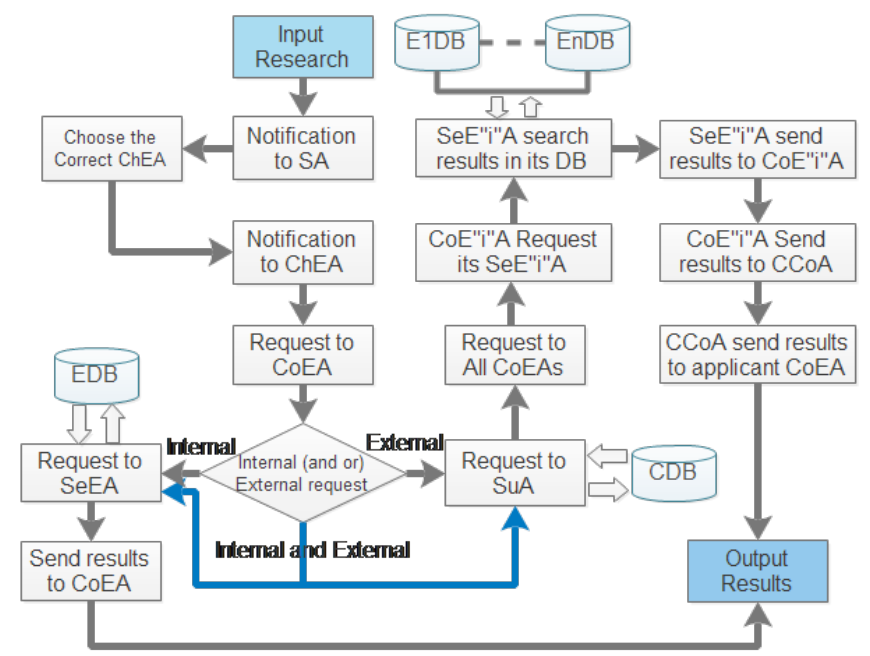

Fig. 11. General process of information retrieval.

Each company named "i" has its proper database (EiDB). When the user initiates the search (Input Research) from his own account, a notification is then sent to the agent CChA. The CoEA agent directs the request both to the external stream (to $\mathrm{SuA}$ ) and the internal stream (to SeEA). SuA is then responsible for sending a message to the other CoEAs to initiate the search for all the necessary confidentiality information in their respective databases. The result of this search makes it possible to return solutions to the agent $\mathrm{SuA}$ which will propagate them to the CoEA that is identified as the requestor.

\section{EXPERIMENTS}

This paper describes the major experiments that are done to demonstrate the feasibility of our suggested approach. For that a simulation study was launched on the basis of Java and JADE platform for multi-agents; Html5, Css3, AJAX and Php for social network.

During the simulation, we generate some databases that are implemented using MySQL. The agents communicate using the JADE platform, which includes several predefined performatives communication. In this work, Sniffer Agent provided by JADE was employed to monitor the communication among agents on the agent platform.
We illustrated three enterprises and 30 connected employers. JADE platform shows containers created for each enterprise (sbo, ametal, inotis) and main container for central agents as it appears in figure 12.

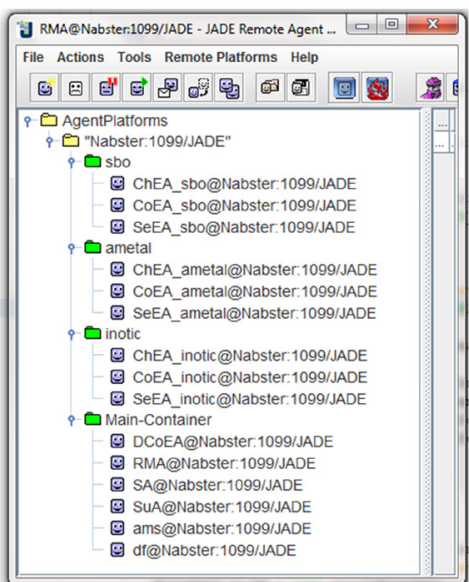

Fig. 12. JADE Platform showing main and enterprise containers.

Figure 13 shows a form which helps a simple user for adding diagnosis or failure solution to his personal catalog.

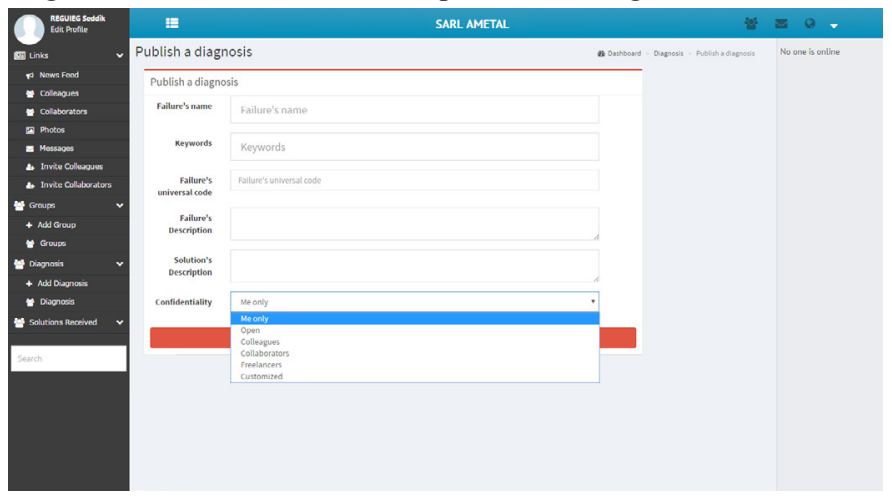

Fig. 13. Form for publishing and adding a diagnosis to a catalog.

Figure 14 gives an overview on the diagnosis' catalog for a user of an enterprise with his confidentialities.

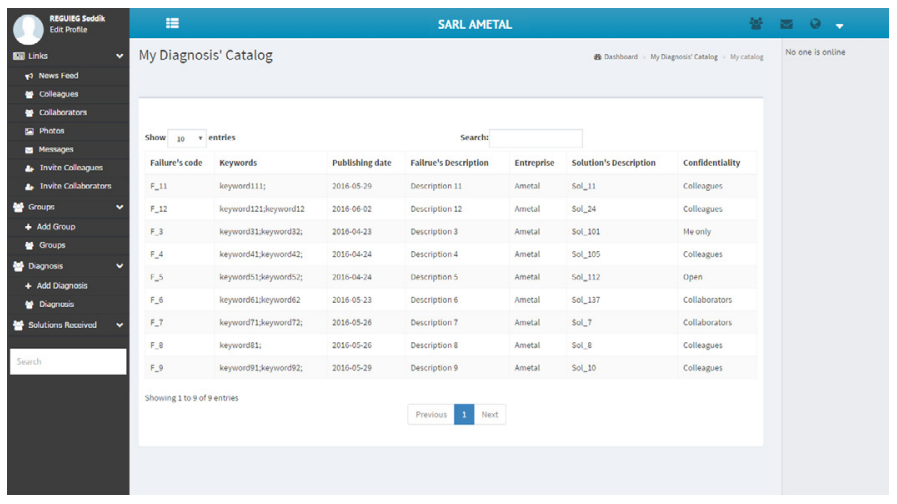

Fig. 14. User's diagnosis' catalog.

Figure 15 shows JADE's sniffer agent which describes the exchange of messages between agents from user's request until finding solutions. 


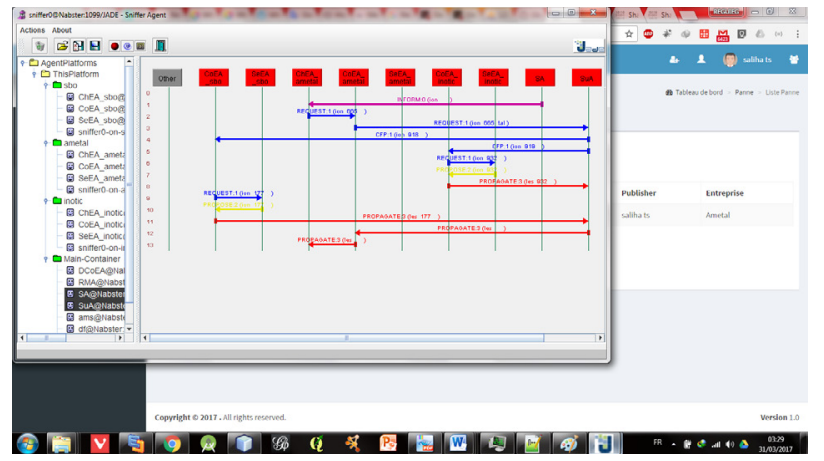

Fig. 15. Sniffer Agent for monitoring messages' exchange between agents.

Figure 16 visualizes some solutions that are received after seeking information from several users coming from different enterprises.

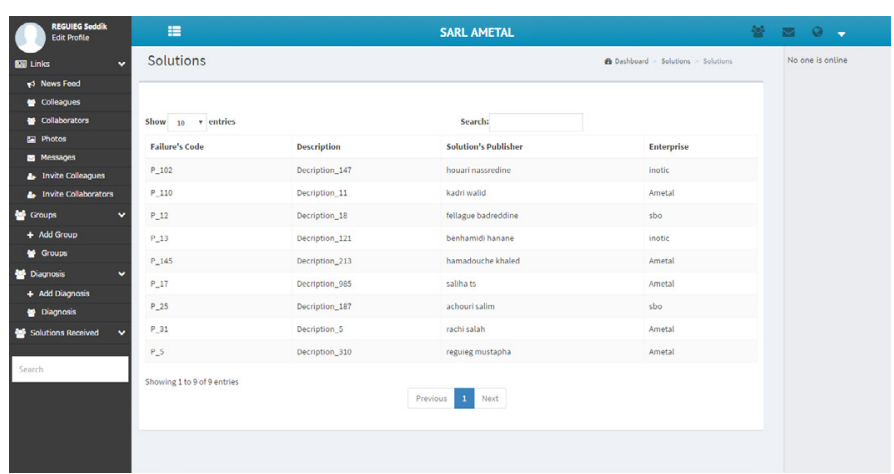

Fig. 16. Solutions received from several users.

\section{Results}

We plot several aspects of the results in series of figures. First, we consider an evaluation of our multi-agents system (MAS). Then, we present a simple comparison between using a webserver and its combination with MAS. Secondly we present an evaluation of our interaction protocol, and finally an evaluation of our social network is given by using a questionnaire.

\section{A. Multi Agents and Webserver Evaluation}

As we had described in previous sections, the adoption of multi agents system decreases the webserver overhead.

Table 4 illustrates the ratio between number of enterprises which adhere in our platform and number of agents created. As we see number of agents increase proportionally with number of enterprises. The messages are generated considering one user's request.

TABLE IV. Ratio Between Number of ENTERPRises, Number of GeNerated Agents and Generated Messages

\begin{tabular}{ccc}
\hline $\begin{array}{c}\text { Number of } \\
\text { Enterprises }\end{array}$ & $\begin{array}{c}\text { Number of } \\
\text { generated agents }\end{array}$ & $\begin{array}{c}\text { Number of } \\
\text { generated messages }\end{array}$ \\
\hline 2 & 8 & 9 \\
10 & 32 & 41 \\
50 & 152 & 201 \\
100 & 302 & 401 \\
200 & 602 & 801 \\
300 & 902 & 1201 \\
600 & 1802 & 2401
\end{tabular}

Table 5 and Figure 17 provide an indication to measure the performance of the web server by considering the contribution of MAS. For that, we had developed our approach with and without using MAS. As we notice, for a single user request, the overhead of the web server which is expressed by the number of queries that are performed using the agents keeps its stability, whatever the number of companies that adhere to our platform. Whereas if we ignore this contribution, the webserver is overloaded by all executed queries. We can explain that all other treatments of research information and respect for confidentialities are made by agents.

TABLE V. COMPARISON BETWEen Number of EXecuted Queries With/ WITHOUT USING MAS

\begin{tabular}{c|c|cc}
\hline \hline & $\begin{array}{c}\text { Approach 1: simple } \\
\text { webserver application }\end{array}$ & $\begin{array}{c}\text { Approach 2: Merging webserver } \\
\text { application and SMA }\end{array}$ \\
\hline $\begin{array}{c}\text { Number of } \\
\text { Enterprises }\end{array}$ & $\begin{array}{c}\text { Number of } \\
\text { webserver's queries } \\
\text { without MAS }\end{array}$ & $\begin{array}{c}\text { Number of MAS } \\
\text { queries }\end{array}$ & $\begin{array}{c}\text { Number of } \\
\text { webserver's queries } \\
\text { using MAS }\end{array}$ \\
2 & 118 & 13 & 105 \\
10 & 158 & 53 & 105 \\
50 & 358 & 253 & 105 \\
100 & 608 & 503 & 105 \\
200 & 1108 & 1003 & 105 \\
300 & 1608 & 1503 & 105 \\
600 & 3108 & 3003 & 105
\end{tabular}

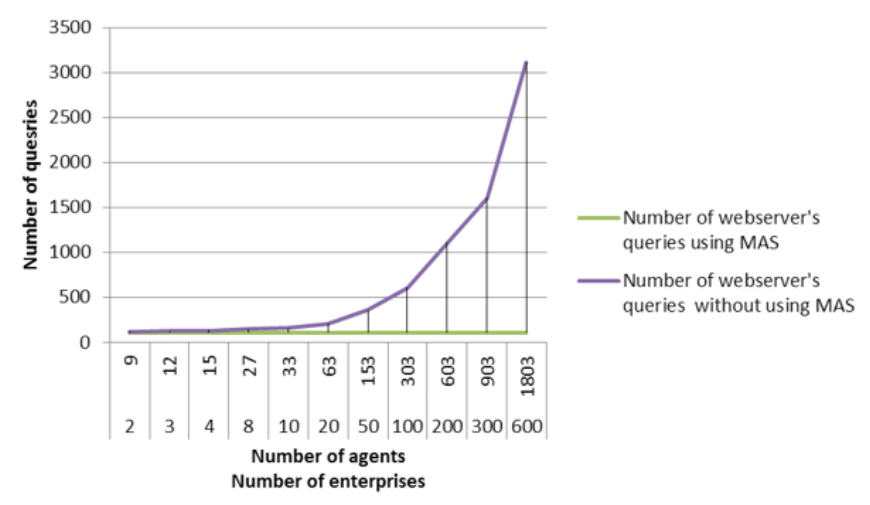

Fig. 17. Number of executed queries with/without using MAS.

\section{B. Social Network Evaluation}

An evaluation of the quality of the developed social network was carried out using a questionnaire. We developed our questions according to several criteria for the ergonomic evaluation of the multiuser interface. The questionnaire was distributed to 30 users of three enterprises.

Figure 18 shows the usability results obtained from the questionnaire, in which a question is generally followed by an inverse question to reveal opposite facts. For each question we assigned a weight. At the end, we count the sum of weights to find the level of satisfaction of the user. Some of the questions in the questionnaire are given in the following tables (Table 6, Table 7, and Table 8).

TABLE VI. Some Relevant Questions RELATED to ACCESSIBILITy

\begin{tabular}{cc} 
Number & Question \\
Q1 & Is content structurally separated from navigational elements? \\
Q2 & Is the website cross-browser compatible? \\
Q3 & Is the website adapted for every computer resolution? \\
Q4 & Is the URL short and simple? \\
Q5 & Is the time of loading the home page correct? \\
\hline
\end{tabular}



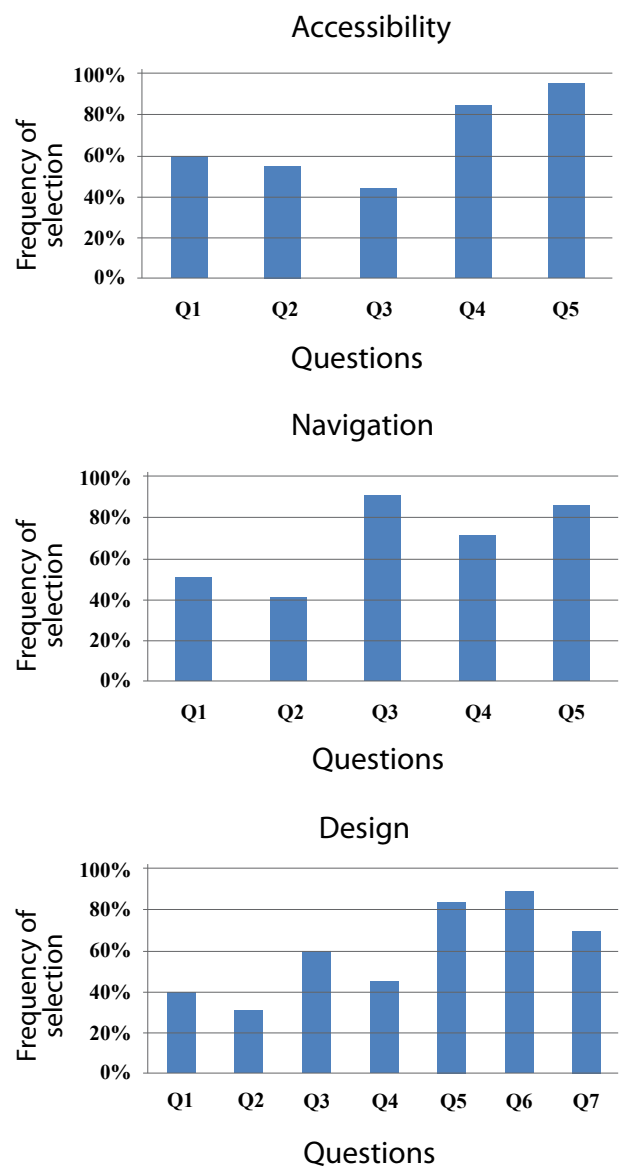

Fig. 18. Statistics of the responses of our platform's users.

TABLE VII. Some Relevant Questions Related to Design

\begin{tabular}{cc}
\hline Number & Question \\
\hline Q1 & Is the site's design aesthetically appealing? \\
Q2 & Are the colors used harmonious and logically related? \\
Q3 & $\begin{array}{c}\text { Are the color choices visually accessible? (For example high } \\
\text { enough in contrast to assist the colorblind and visually impaired } \\
\text { in reading the site appropriately) }\end{array}$ \\
Q4 & $\begin{array}{r}\text { Is the design audience appropriate?- The standard text size should } \\
\text { be readable for visitors who don't know how to adjust their }\end{array}$ \\
Q5 & Are the fonts easy to read on various screen resolutions? \\
Q6 & The site is homogeneous from one page to another (no visual break) \\
Q7 & The number of colors is limited (correct $=4) ?$
\end{tabular}

TABLE VIII. Some ReLEVANt Questions Related to NAVIGATION

\begin{tabular}{|c|c|}
\hline Number & Question \\
\hline Q1 & $\begin{array}{l}\text { Are links labeled with anchor text that provides a clear indication } \\
\text { of where they lead without over using exact match anchor text? }\end{array}$ \\
\hline Q2 & $\begin{array}{l}\text { What is the maximum number of clicks it takes to reach a page } \\
\text { within the depths of the site? }\end{array}$ \\
\hline Q3 & $\begin{array}{l}\text { Is a response given immediately ( } 0.1 \text { seconds) after a click is } \\
\text { made on a hyperlink? }\end{array}$ \\
\hline Q4 & Do clickable items stylistically indicate that they are clickable? \\
\hline Q5 & $\begin{array}{c}\text { How intuitive is it to navigate? Are signs obvious or obscured? } \\
\text { Buttons/Links Like Text that are not clickable and vice versa, } \\
\text { links/buttons that cannot be identified as such? }\end{array}$ \\
\hline
\end{tabular}

We proposed a questionnaire consisting of six (6) categories for thirty (30) adhered users. We collected the answers to the questions and we obtained the results that are presented in Figure 19.

Satisfaction rate of 30 users

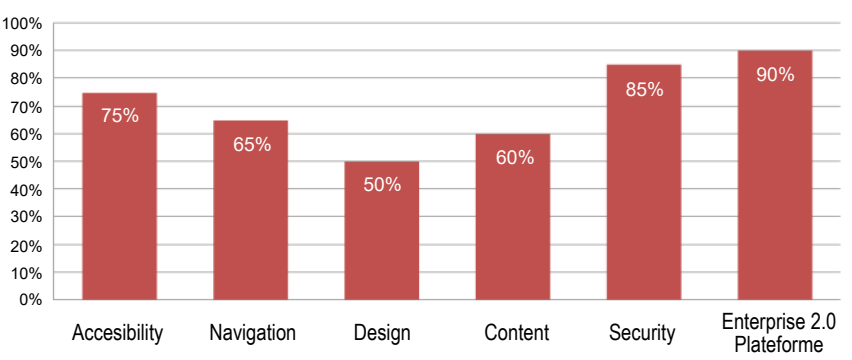

Fig. 19. Evaluation of the usability of our platform.

According to the results, we concluded that the system could be improved to increase design and content. This can be achieved by adding new collaborative environmental assessment questions.

\section{CONCLUSION AND FUTURE WORKS}

The emergence of Web 2.0 and its recent success in publicsector organizations (Enterprise 2.0) has served to fulfill objectives that are planned for an organization. It is shown in this research that the application of an enterprise 2.0 project (that is based on Web 2.0 technologies) could assist organizations improve business processes and increase employee's productivity, communications, and information sharing.

More precisely, the emergence of Enterprise 2.0 platforms has given SMEs a big step forward in providing solutions to overcome hard-tosolve problems. In this paper, some of them have been tackled especially collective intelligence, social interaction, and knowledge management. Our suggested solution implies three domains of research: enterprise 2.0, multi-agents systems (MAS), and social network.

Our motivation behind the choice of the MAS is to lighten the Web server tasks in enterprise 2.0 platform, and to provide more security to information exchange with some respect to confidentialities intra and inter enterprises. The objective of our work is to propose a helpful approach utilizing new coordination protocol (CordiNet) and computational collective intelligence for enterprise 2.0 design. Firstly, we implement a collaborative environment (Pro Social Network) that allows employers to share diagnosis and fault repair procedures. Secondly, we propose a coordination environment that is based on multi-agents system and interaction protocol.

This study is based on capitalization of user's intelligence that is expressed in catalogs in order to be used by other users with a specialized authorization. The MAS is responsible of managing information traffic inter or intra enterprises. To optimize and reorder the information research, a max cost uniform algorithm has been used with a slight modification.

Some companies are candidates and interested by our prototype such as: Unilever (Oran-Algeria), Inotis (Oran-Algeria) for a real implementation.

For further research, this framework should be improved by using learning coordinator agent which capitalizes knowledge from past experiences. Furthermore, even if our framework consists on a learning feature to find solution, a semantic feature is very essential. We intend to integrate some tools that treat the Semantic Web. 


\section{REFERENCES}

[1] A. P. McAfee. "Enterprise2.0: The Dawn of Emergent Collaboration". MIT Sloan Management Review, 2006.

[2] T. O'Reilly, "What is web 2.0: Design patterns and business models for the next generation of software". Communication \& Strategies 1, 2007.

[3] A. Sheun, "Web 20: A strategy guide : Business thinking and strategies behind successful web 2.0 implementations". O’Reilly Media, 2008.

[4] D. Beer, R. Burrows, "Sociology and, of and in web 2.0: some initial consideration". Sociological Research Online 12, 2007.

[5] R. L. Cross, and A. Parker, "The Hidden Power of social networks: Understanding How Work Really Gets Done in Organizations". Harvard Business Press. Boston, MA:USA, 2004.

[6] J. Payne, "Using wikis and blogs to improve collaboration and knowledge sharing". Strategic HR Review 7, 2008, pp. 5-12.

[7] A. M. Zhang, Y. Zhu, H. Hildebrandt, "Enterprise networking web sites and organizational communication in Australia". Business Communication Quarterly 72, 2009, pp. 114-119.

[8] P. Lévy, "L'intelligence collective : pour une anthropologie du cyberspace", la Découverte, 1997.

[9] D. Billouard-Fuentes, "Emergence d'Intelligence Collective dans un Environnement Numérique de Travail", 7ème colloque du chapitre français de l'ISKO International Society for Knowledge Organization, Lyon, France, pp.10, June 2009.

[10] E. Lazega, "Analyse de réseaux et sociologie des organisations". Revue française de sociologie, 1994, pp. 35-23.

[11] R. Sikora, and M.J. Shaw, "A multi-agent framework for the coordination and integration of information systems". Management science, vol 44, 1998, pp.65-78.

[12] T. Blecker, and G. Graf, "Multi-agent systems in internet based production environments - an enabling infrastructure for mass customization". Paper presented at the second Interdisciplinary World congress on Mass Customization and Personalization Munich, Germany. In proceeding, 2003, pp, 1-27.

[13] S. Liu, and R.I.M. Young, "Utilizing information and knowledge models to support global manufacturing coordination decisions". Int. J. of computer Integrated Manufacturing, Vol. 17, 2004, pp.479-492.

[14] P.M. Francisco, and H.N. Douglas, "Multi-agent mediator architecture for distributed manufacturing", J. of Intelligent Manufacturing, Vol. 7, 1996, pp. $257-270$.

[15] D. Wang, S.V. Nagalingam, and G.C.I. Lin, "Development of an agentbased Virtual CIM architecture for small to medium manufacturers", Robotics and Computer-Integrated Manufacturing, Forthcoming, doi.10.1016/j.rcim.2005.09.001.

[16] L. Wahl and A. Kitchel, "Internet based collaboration tools", In International journal of e-Collaboration, Vol 12, Issue1, January-march 2016.

[17] H.N. Sad and N. Taghezout. "Integrating Agents into a Collaborative Knowledge-based System for Business Rules Consistency Management" In International Journal of Interactive Multimedia and Artificial Intelligence, Vol $4 \mathrm{~N}^{\circ}$ 2, pp 61-72, December 2016.

[18] E. Franchi "A multi-Agent Implementation of Social Networks", CEUR Workshop Proceedings, 2010, vol 621.

[19] I. Bessedik and N. Taghezout : "Web 2.0 Integration in a WEB-Based Decision Support System: Effect Study of Social Networking on DecisionMaking“" In IJWA, Vol 4, N², pp 69-77, (2012).

[20] N. Flor, D. Piraquive, R. G. Crespo and Víctor H. M. García. "Managing Projects and Organizations within Enterprise 2.0“, Pensee Journal Vol 76 No. 5; May 2014.

[21] M. Corso, A. Martini and Andrea Pesoli, "Enterprise 2.0 : What models are emerging?" The results from a 70 case-based research, In International journal of Knowledge and Learning 4(6), pp: 595-612, January 2008.

[22] N. Ramadan and N. Al-Qirim, "New Hybrid Web 2.0 Adoption Governance Framework for Public Sector", In International journal of e-Collaboration 11, 1, pp 8-21, January-march 2015.

[23] B. Schauer, M. Zeiller, and R. Matzinger, "Open source tools for enterprise 2.0: A Feature-Based Adaptable Analysis". A. Fred et al. (Eds): IC3K 2011, Springer-Verlag Berlin Heidelberg. CCIS 348, pp. 407-422, 2013.

[24] K. Riemer, "The Market for E-Collaboration Systems - Identification of System classes using cluster analysis". In Österle, H. Schelp, J., Winter,
R. (eds.) Proceeding Fifteenth European Conference on Information Systems, 2007, pp. 346-357.

[25] J. Xu, J. Zhang, T.Harvey, J. Young, "A survey of Asynchronous Collaboration Tools". Information Technology Journal 7(8), 2008, pp. 1182-1187.

[26] J. Soriano, D. Lizcano, M. A. Canas, M. Reyes, and J. J. Hierro. "Fostering Innovation in a MAshup-oriented Enterprise 2.0 Collaboration Environment". System and Information sciences Notes, no 1, 2007, pp. 62-68.

[27] D. Bianchini, V. De Antonellis, M. Melchiori, "Collaborative mashup development in Enterprise 2.0". Proceedings of the Fifth Interop-Vlab. It Workshop on Complexity of Systems, Complexity of Short Papers for Seminar IM. Volume 2013.

[28] J.L. KONING and S. PESTY, "Modèles de communication, in Principes et architectures des systèmes multi-agents", édité par Jean-Pierre Briot et Yves Demazeau, Collection IC2, Hermes Science Publications, Paris, 2001.

[29] Gray RS., et al., "Mobile-agent versus client/server performance: scalability in an information-retrieval task". Mobile Agents: 5th International Conference, MA, Atlanta, Georgia, number 2240 in Lecture Notes in computer Science, 2001, pp. 229-243.

[30] The FIPA ACL Message Structure Specifications. http://www.fipa.org/ specs/fipa00061/, 2002.

[31] P.M. Francisco, and H.N. Douglas, "Multi-agent mediator architecture for distributed manufacturing", J. of Intelligent Manufacturing, Vol. 7, 1996, pp.257-270.

[32] T. D. Wilson, "Human information behavior". Information Science Research 3, 2000, pp. 49-55.

[33] D. O. Case, "Looking for Information, Second Edition: A Survey of Research on Information Seeking, Needs and Behavior", Library and Information Science, academic Press, December 2006.

[34] Fisher, K. E., S. Erdelez, and L.E. Mckechine. "Theories of Information Behaviour". Asist Monograph. Information Today, August, 2005.

[35] X. Yun and C. Jianbin. "A Self-organization Analysis of Knowledge Collaboration with Enterprise 2.0 Application". In International Journal of u- and e- Service, Science and Technology Vol.8, No.1, 2015, pp.211-220.

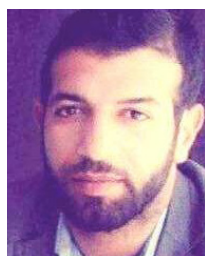

\section{Seddik Reguieg}

Seddik Reguieg was born on Dec 5, 1985 in Oran (Algeria); he earned his Computer Science Engineering Degree with specialization Industrial Computer Science in 2008 from Oran 1 Ahmed Ben Bella University (Algeria), he also received Computer Science Magister Degree with specialization in Diagnostic and Decision-Making Assistance and Human Interaction Machine in 2011 from the same university. He is currently an assistant teacher at University of Hassiba Benbouali of Chlef and a Ph.D. student in Computer Science Department at University of Oran 1 Ahmed BenBella. His fields of special interest include Artificial Intelligence, Collaborative filtering, Recommendation Systems, WEB 2.0 Technologies, and Graph theory.

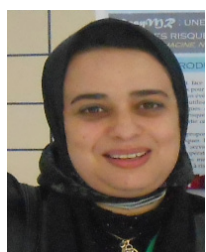

Noria Taghezout

Noria Taghezout is an assistant professor at university of Oran 1 Ahmed Ben Bella, Algeria. She holds her doctorate thesis in MITT at PAUL SABATIER UNIVERSITY in France in 2011. She also received another doctorate thesis in Distributed Artificial Intelligence from university of Oran 1 Ahmed Ben Bella in 2008. She holds a Master degree in Simulation and Computer aided-design. She conducts her research at the LIO laboratory as a chief of the research group in Modeling of enterprise process by using agents and WEB technologies. Since she studied in UPS Toulouse, she became a member of the EWG-DSS (Euro Working Group on Decision Support Systems). She is currently lecturing Collaborative decision making, Enterprise management and Interface human machine design. Her seminars, publications and regular involvement in Conferences, journals and industry projects highlight her main research interests in Artificial Intelligence. 